\title{
An Early Diagnosis of Gastroepiploic Arterial Aneurysm during a Routine Abdominal Ultrasound Study
}

\author{
Giovanni Casella, ${ }^{1}$ Adriana Sartirana, ${ }^{2}$ Pietro Vandoni, ${ }^{3}$ Camillo Di Bella, ${ }^{4}$ \\ Fabio Pagni, ${ }^{4}$ and Paolo Nobili ${ }^{5}$ \\ ${ }^{1}$ Medical Department, Desio Hospital, Piazza Benefattori, Monza e Brianza, 120033 Desio, Italy \\ ${ }^{2}$ Radiology Department, Desio Hospital, Desio, Monza e Brianza, Italy \\ ${ }^{3}$ Cardiology Department, Desio Hospital, Desio, Monza e Brianza, Italy \\ ${ }^{4}$ Pathology Department, Desio Hospital, Desio, Monza e Brianza, Italy \\ ${ }^{5}$ Vascular Surgery Department, Clinica "San Giuseppe", Milan, Italy \\ Correspondence should be addressed to Giovanni Casella, petala.83@tiscali.it
}

Received 14 May 2011; Accepted 13 June 2011

Academic Editors: C. Elsing and L. H. Lai

Copyright ( 2011 Giovanni Casella et al. This is an open access article distributed under the Creative Commons Attribution License, which permits unrestricted use, distribution, and reproduction in any medium, provided the original work is properly cited.

Gastroepiploic arterial aneurysm (GEAA) is a rare condition, but the rupture risk is very high. We report the case of a patient with incidental finding of GEAA during US examination. The diagnosis was confirmed by a computed tomography and an angiographic study. A classic laparotomy with aneurysmectomy has been successfully performed.

\section{Case Report}

Gastroepiploic arterial aneurysm (GEAA) is a rare condition, but the rupture risk is very high [1] because in $90 \%$ of the affected patients it is shown as a vascular emergency [1]. Visceral arterial aneurysms are rare and their incidence in the general population has been reported at $0.01-2 \%$ [2] We describe a diagnosis of unruptured gastroepiploic artery aneurysm by (thank) a routine ultrasound diagnosis. We observed, in June 2005, a-64-year old Caucasian man, HbsAg "carrier" with consecutively normal liver enzymes. During a routine ultrasound examination, we noted an abnormal circular anechogenic area of $2.5 \mathrm{~cm}$ in diameter in the epigastric region, near the visceral peritoneal layer. A Doppler study revealed an arterial pulse suggesting the hypothesis of an arterial aneurismatic dilation. This hypothesis has been confirmed by a computed tomography (CT) and an angiographic study (Figure 1), and we concluded for right GEAA without evidence of other splachnic aneurysms. Nonsurgical treatment as transcatheter arterial embolization (TAE) has been considered, but it was impossible to perform for a long and tortuous vessel's anatomy. A classic laparotomy with aneurysmectomy has been successfully performed.
Histologic examination and absence of blood-antineutrophil Cytoplasm antibodies (P-ANCA) ruled out polyarteritis nodosa (PAN). The patient, at present after 5 years, is in good general condition. Stanley and Zelenoch [2] reported a study about more than 3000 visceral arterial aneurysms [2](this data is confirmed); $60 \%$ are splenic, $20 \%$ of the hepatic artery, $5.5 \%$ of the (mesenteric) superior mesenteric artery, $4 \%$ of the celiac axis, $2 \%$ of the pancreatic duodenal artery and its branches, $1.5 \%$ of the gastroduodenal artery, and less than $1 \%$ of the inferior mesenteric artery. Pulli et al. [3], in a series of 55 patients affected by visceral artery aneurysm, reported only 1 case of right gastroepiploic aneurysm (1.7\%). These GAAs are mostly common in 50-60 years old men, often affected by arterial hypertension and atherosclerosis [2]. Other etiologic factors are infection, medial necrosis, trauma, pregnancy, portal hypertension, biliary disease, pancreatitis, and connective tissue disease [2]. Clinical manifestations of gastrointestinal arterial aneurysmes (GAA) vary from free of symptoms to epigastric pain. Pulli et al. [3] reported that the diagnosis was incidentally made during ultrasound examination performed for unrelated abdominal disease in 52 of all 55 patients with visceral artery aneurysm 

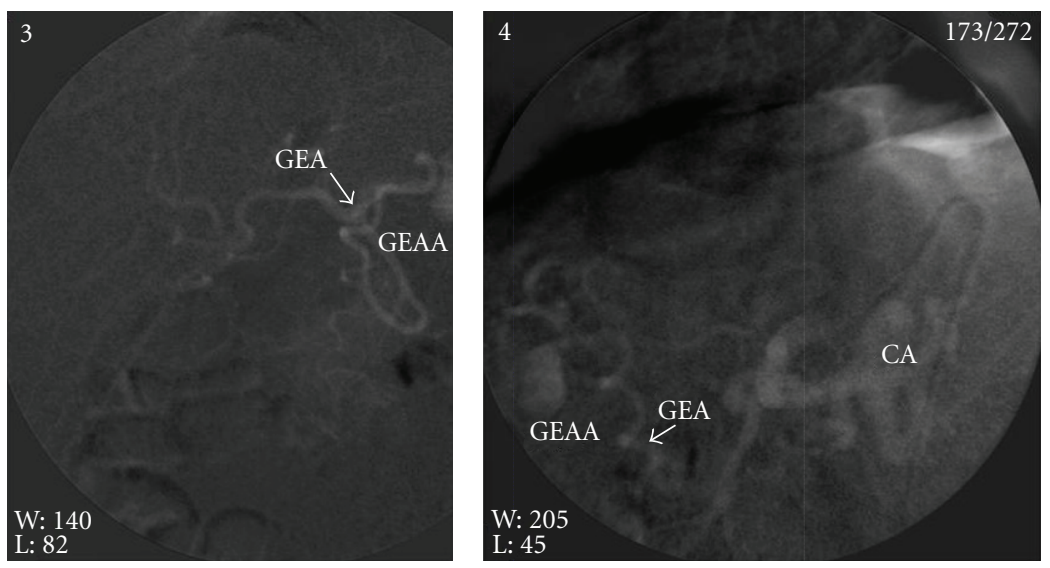

Figure 1

(94.6\%) [3]. Up to $50 \%$ of patients remain asymptomatic before the aneurysm rupture and operative mortality is $50-70 \%$ for emergency procedures versus $0-3 \%$ for elective surgery [3]. The widespread use of "Imaging" devices permits an early diagnosis in asymptomatic patients allowing an "elective" surgical therapy [3]. About $81 \%$ of the GEAAs are diagnosed after the rupture [4]. The mortality of these aneurysms after rupture is very high [4]; aneurysmectomy is mandatory if the diagnosis has been performed before the rupture [4]. Angiography should be performed in all patients with single aneurysm because $15-40 \%$ of these have multiple aneurysms as the cases reported by Pulli et al. [3] that showed multiple visceral artery aneurysm (VAA). Borioni et al. [4] reported that this can be eliminated. Celiac arteriography (CA) permits a correct diagnosis in $44.4 \%$ of all patients studied, surgery in 50\% of the patients, and CT with medium contrast only in 5.6\%. This low diagnostic level of CA could be due to the slow flow of blood in this tract that it cannot be visualized by CA [4]. Surgical excision may be performed by traditional surgery or by laparoscopic surgery [4]. Recently, trancatheter arterial embolization (TAE) -no (TACE) with an occlusion of the aneurysm should be always considered before every surgical procedure [5].

\section{References}

[1] M. De Angelis, C. Vogel, B. Horowitz et al., "Ruptured gastroepiploic aneurysm," Journal of Clinical Gastroenterology, vol. 18, no. 3, pp. 261-262, 1994.

[2] J. C. Stanley and G. B. Zelenoch, "Splachnic artery aneurysms," in Vascular Surgery, R. B. Rutherford, Ed., pp. 1124-1139, Saunders, Philadelphia, Pa, USA, 1995.

[3] R. Pulli, W. Dorigo, N. Troisi, G. Pratesi, A. A. Innocenti, and C. Pratesi, "Surgical treatment of visceral artery aneurysms: a 25year experience," Journal of Vascular Surgery, vol. 48, no. 2, pp. 334-342, 2008.

[4] R. Borioni, M. Garofalo, P. Innocenti et al., "Hemoperitoneum due to spontaneous rupture of an aneurysm of the left gastroepiploic artery," Journal of Cardiovascular Surgery, vol. 40, no. 1, pp. 63-64, 1999.
[5] N. Tulsyan, V. S. Kashyap, R. K. Greenberg et al., "The endovascular management of visceral artery aneurysms and pseudoaneurysms," Journal of Vascular Surgery, vol. 45, no. 2, pp. 276283, 2007. 


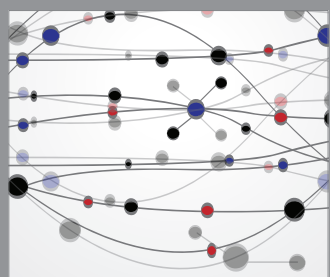

The Scientific World Journal
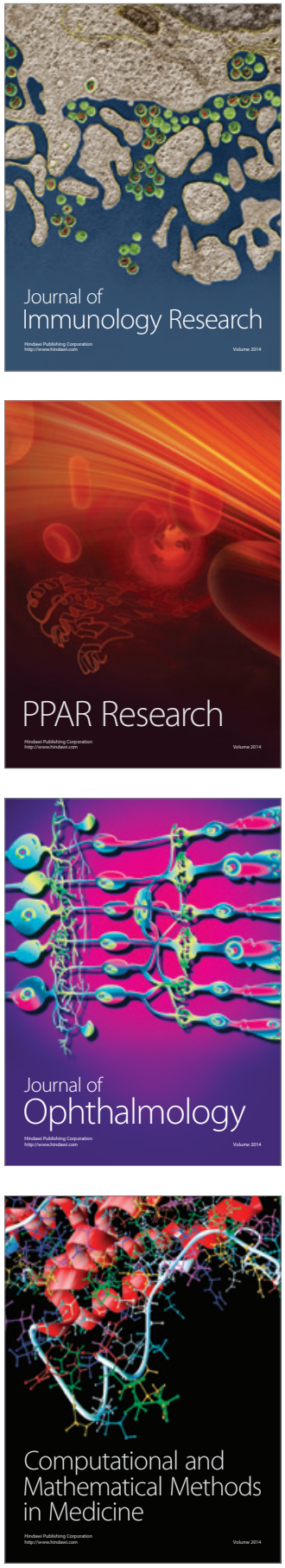

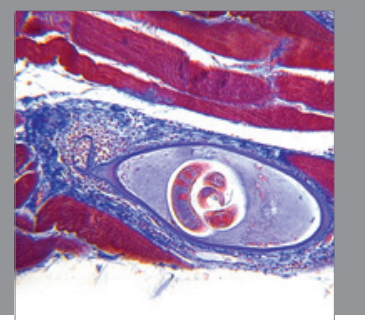

Gastroenterology

Research and Practice
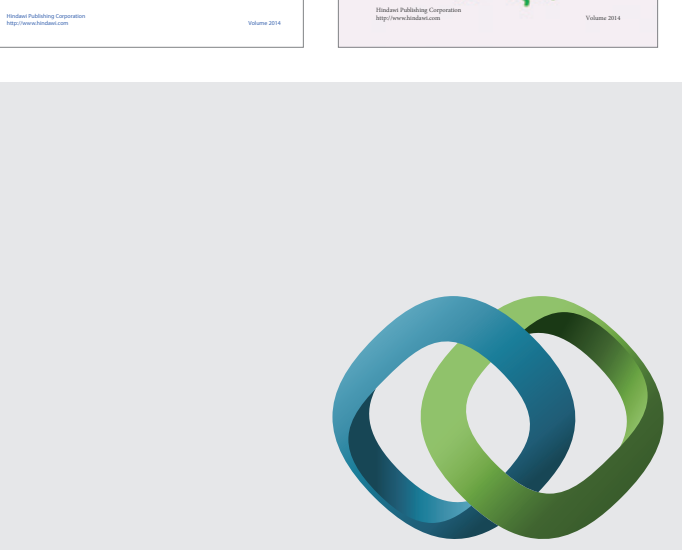

\section{Hindawi}

Submit your manuscripts at

http://www.hindawi.com
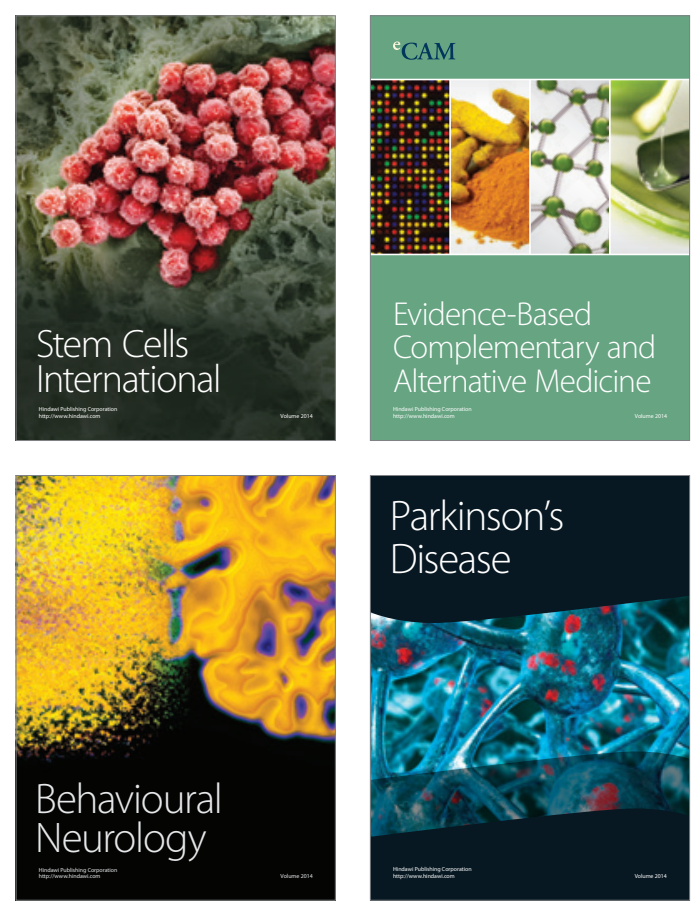

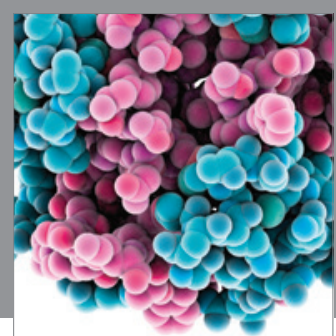

Journal of
Diabetes Research

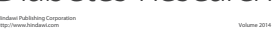

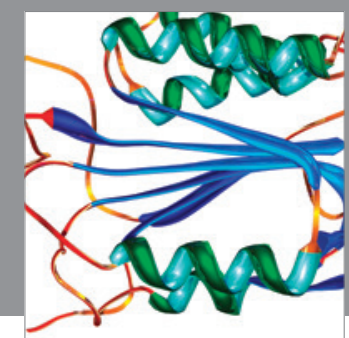

Disease Markers
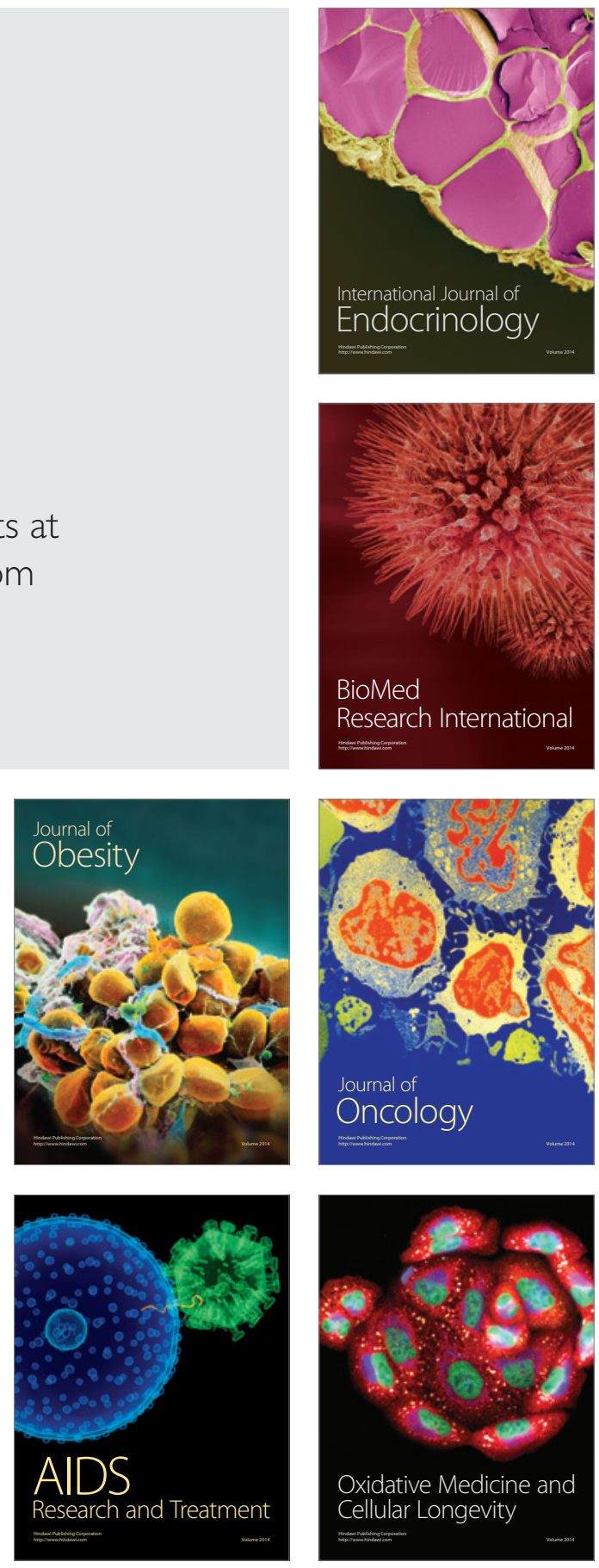